\title{
Study on Clinical Nursing of Extravasation of Chemotherapeutic Drugs Treated with Taiyi Cream and Dexamethasone
}

\author{
Yunfei Jiang, Xiuli Chen, Fei Lin
}

The Third Affiliated Hospital of Qiqihar Medical University, Qiqihar, Heilongjiang, 161000

Keywords: clinical nursing; Extravasation of Chemotherapeutic Drug; Dexamethasone

\begin{abstract}
In order to observe and analyze the efficacy and adverse reactions of Taiyi cream and dexamethasone topical application on chemotherapeutic drug-induced phlebitis in elderly patients, and provide reference for more effective treatment of chemotherapeutic drug extravasation in elderly patients, 50 elderly patients with phlebitis caused by extravasation of chemotherapy drugs were included in the study from March 2015 to March 2016. They were divided into control group and experimental group according to the order of admission. There were 25 patients in each group. The group was treated with Taiyi ointment and externally applied dexamethasone, and the control group was given magnesium sulfate externally. The efficacy and adverse reactions of the two groups were observed. The effective rate of treatment in the experimental group (96.0\%) was significantly higher than that in the control group (76.0\%), $\mathrm{P}<0.05$. The 72-hour cure rate in the experimental group was significantly higher than that in the control group $(\mathrm{P}<0.05)$. There was no adverse reaction in both groups. Taiyi cream combined with dexamethasone topical application is more effective than conventional magnesium sulfate in elderly patients with phlebitis caused by chemotherapy drug extravasation. It is economically safe and effective, with no obvious adverse reactions, and has significant clinical application value.
\end{abstract}

\section{Introduction}

Chemotherapy is one of the important means for the treatment of malignant tumors. In the course of intravenous infusion of chemotherapy drugs, extravasation of chemotherapy drugs is one of the most common clinical complications. The incidence of chemotherapeutic drug extravasation is reported to be $0.1 \%$ to $6.0 \%$ in the country and $5.0 \%$ in foreign reports [1]. The effect of extravasation on the body varies with the type of drug and the amount of drug that leaks. Extravasation caused severe damage not only related to the direct effect of the drug, but also related to the solubility of the drug, the $\mathrm{pH}$ of the solution, etc. [2]. If improperly handled, it can cause redness, swelling and pain in the local tissue. In severe cases, it requires surgical debridement and skin grafting. It not only causes great pain to patients, increases the cost of treatment, and affects the implementation of follow-up treatment plans. It directly affects the quality of life of patients, and may even cause disputes between doctors and patients. The methods for prevention of extravasation of chemotherapy drugs, related care after extravasation, and research directions are reviewed.

\section{Materials and Methods}

Patients with phlebitis caused by extravasation of chemotherapeutic drugs from March 2015 to March 2016 were included in the study, including 23 males and 27 females. All patients were over the age of 60 , aged from 60 to 74 years, and the average age. $66.8 \pm 5.3$ years old, Chemotherapy drug extravasation area is $3.0 \times 3.5 \mathrm{~cm}$ or more, according to phlebitis criteria: I degree (injection of local pain) in 26 cases, II degree (inflammation of blood vessels and surrounding tissues) In 13 cases, there were 11 cases of degree III (local swelling and blisters and necrosis, no skin ulceration). According to the order of admission, the patients were divided into control group and test group. There were 25 patients in each group. There was no significant difference in general data (gender, age, phlebitis, etc.) between the two groups, $\mathrm{P}>0.05$. , Comparability between groups.

The experimental group chose Taiyi ointment (produced by Zhoukou Bio-Chemical Co., Ltd., 
Zhoukou, Henan Province). Each time, 2 sticks of heat were selected to make a paste, and after cooling slightly, dexamethasone $5 \mathrm{mg} / \mathrm{ml} 2$ sticks were added and mixed thoroughly. The drug is extravasated for 24 hours. The area is slightly larger than the lcm around the affected area, and the thickness is 1 to $2 \mathrm{~mm}$. The tissue is covered with tissue paper. After wrapping with a plastic wrap, the cling film is wrapped with a $40-50^{\circ} \mathrm{C}$ hot towel, and then the fresh-keeping film is used. The membrane is wrapped in a hot towel and the hot towel is cooled to re-wet the affected area. Avoid burns. The next day after the replacement of the skin to replace the local, usually 2 to 3 days for a course of treatment, severe 1 week for a course of treatment. Patients in the control group were given 25\% magnesium sulfate gauze (4 to 6 layers) with local wet compress. The area was slightly larger than the lcm around the affected area. Then cover the warm towel with plastic wrap, and then rehydrate the affected area with hot towel cooling, every hour. Replace it once and keep the magnesium sulfate gauze moist.

Observed indicators and evaluation of curative effect Comparison of the efficacy and adverse reactions of the two groups of patients. According to the standards established by the United States intravenous infusion care (INS), classification is based on the most severe symptoms. The skin pain was calculated according to a 10-point scale. The degree of skin swelling was grade 3 light to medium weight. Efficacy evaluation: 1 Healing: Localized swelling and heat pain disappeared, the lump became soft, the dry blisters fell off, and the epithelium regenerated. 2Effect: Local swelling and heat pain was relieved, blisters dry; 3 Ineffective: local swelling and heat pain did not ease, even worsened, the leeches fuse into flakes. The total effective rate is the sum of recovery and effectiveness. SPSS 19. 0 was used to process the count data and measurement data obtained. The $t$ data was used for the measurement data and the chi-square test was used for the count data. When $\mathrm{P}$ $<0.05$, there was a significant difference, which was statistically significant.

The therapeutic effect of the two groups was $96.0 \%$ compared with the experimental group, which was significantly higher than the $76.0 \%$ of the control group. The difference was statistically significant $(\mathrm{P}<0.05)$. The 72 -hour recovery rate in the two groups was significantly higher than that in the control group at 72 hours $(\mathrm{P}<0.05)$. There was no adverse reaction in both groups, suggesting that the Taibai ointment and dexamethasone topical application is safe and effective in the treatment of extravasation of chemotherapy drugs.

The extravasation of drugs into the blood vessels will cause direct stimuli to the tissues and cause the release of inflammatory mediators. At the same time, they will increase the permeability of the blood vessel's inner wall and cause local tissue acute inflammation [3]. Extravasation of chemotherapeutic drugs can cause local skin pain, swelling or even necrosis, and even amputation. For elderly chemotherapy patients, because of long-term treatment and physical weakness, the probability of extravasation of chemotherapeutic drugs is higher, causing not only physical and psychological trauma to the patient, but also increasing hospitalization costs, affecting the next cycle of chemotherapy, and delaying treatment. The use of $50 \%$ magnesium sulfate wet heat as the most common treatment, through the use of hypertonic effect, promote the regression of tissue edema, to achieve the purpose of pain. However, this method of treatment has limitations. Magnesium sulfate is easily crystallized when it is cold, and dehydration can be aggravated by hypertonic liquid leakage. Therefore, it can only be used for extravasation caused by high vascular permeability [4], and for skin. Irritating, patients often feel uncomfortable. Chinese medicine believes that the venipuncture injury during the infusion process leads to poor blood circulation in the local venous blood, and poisonous evil accumulates in the bloodstream, leading to poor phlebotomy of local venous blood and causing phlebitis. Taiyi ointment has the effect of swelling and clearing fire, detoxification and myogenic activity. It is mainly used to treat acute and chronic purulent skin infections, folliculitis, cellulitis, sputum, acute mastitis, lymphadenitis, multiple abscesses, eczema, and acne infections. And other skin infectious diseases. Dexamethasone is a glucocorticoid that has a stabilizing effect on lysosomal membranes and reduces the release of proinflammatory substances such as bradykinin, serotonin, and histamine, and inhibits allergic inflammation [5]. The combined use of Taizhi ointment and dexamethasone topical treatment of phlebitis caused by docetaxel extravasation, a significant effect, the study found that the treatment 
group was $96.0 \%$ effective rate, 72 hours recovery rate reached $94.7 \%$, were significantly higher than the control group, and no significant adverse reactions. In summary, the effect of tai tai ointment combined with dexamethasone external application on phlebitis caused by chemotherapy drug extravasation is more significant than traditional magnesium sulfate, and it is economically safe and effective without obvious adverse reactions, and has significant clinical application value.

\section{Chemotherapy Drug Extravasation Prevention Methods}

Nursing staff's professional training Nursing personnel who are engaged in intravenous chemotherapy must undergo systematic and professional training before they are on the job. They are skilled in mastering the care points of chemotherapy, the nature of drugs, the classification of irritation, and the training of venipuncture techniques. According to the evaluation of chemotherapy patients, a standardized and scientific chemotherapy care plan was drawn up.

Reasonably select the vein to follow the "Intravenous Care Practice Guidelines and Implementation Regulations (2009)", 1) infusion stimulation chemotherapeutic drugs should alternately use the forearm thick, straight, good elasticity, or choose to stay 2 to $3 \mathrm{~cm}$ away from the joint intravenous administration. 2) Infusion of cyanotic chemotherapy drugs should be performed with central venous catheterization. 3) to avoid ipsilateral upper limb chemotherapy; patients with superior vena cava syndrome to avoid chemotherapy in the upper extremity, the choice of femoral vein catheter.

To master the infusion technique, first establish a vein channel with $100 \mathrm{ml}$ normal saline or $5 \%$ glucose solution, properly fix the needle after successful puncture, observe no leakage, and then infuse the chemotherapy drug; do not use a chemotherapeutic needle to directly puncture the vein or pull needle.

When combining drugs with rational use of drugs, in principle, non-hair loss agents should be input first, and low-concentration drugs should be input first; rapid flushing with isotonic fluid (100 $\mathrm{ml}$ saline or $5 \%$ glucose solution) between two chemotherapy drugs; infusion of chemotherapeutic drugs Concentration should not be too high, the speed should not be too fast, generally infusion rate of $5 \mathrm{ml}$ per minute, once every 3 to 4 min once blood return, to avoid blood vessels in a short period of time by strong stimuli resulting in damage.

Strengthen drug knowledge education. Patients should minimize physical movements such as going to the bathroom when injecting irritating chemotherapy drugs. Infusion of the limbs should not be oppressed, so as not to affect blood return and cause drug leakage. During the treatment and 5 to 7 days after administration, do not apply hot blood to puncture blood vessels. Platinum chemotherapy drugs should be forbidden for cold.

Prophylactic medication Chemotherapy can be routinely applied with Hirudoid ointment along the vein or wetted with magnesium sulphate solution; Zhou Yinping suggested that $2 \%$ lidocaine hydrochloride can be used before entering chemotherapy drugs. Ml + dexamethasone sodium phosphate $5 \mathrm{mg}+$ physiological saline $7 \mathrm{ml}$ diluted slowly intravenous injection; Yan Yongyi et al proposed using ice and aloe vera external application before and infusion chemotherapy; skin nitric acid above the puncture point Glycerine patch; VitC, dexamethasone and other drops from the Murphy's dropper.

Strengthen the inspection of infusion chemotherapy drugs should be closely observed when the return of blood, local pain, extravasation and so on. If extravasation or suspected leakage of chemotherapeutic drugs is found, they are treated according to extravasation.

Extravasation commonly used antidotes 1) dimethyl hydrazine maple: for anthracyclines, mitomycin, cisplatin, ifosfamide, 5-FU and other drugs extravasation; Usage: Each time 50\% to $90 \%$ dimethyl hydrazine Maple solution $(1.5 \mathrm{ml})$ was applied to the extravasation site and used once every 6-8 hours for 7-14 days. 2) Sodium bicarbonate: suitable for detoxification of quinone diterpenoids; Usage: sodium bicarbonate and normal saline mixed into a 1:1 preparation, local injection of extravasation, the total amount does not exceed $10 \mathrm{ml}$, can also be used for local cold compress. 3) Hyaluronidase: suitable for extravasation of vinblastine, high concentrations of podophyllotoxin and paclitaxel; Usage: external subcutaneous injection of $150 \sim 190$ U, repeated 
injections several hours later. 4 sodium thiosulfate: suitable for alkylating agent, high concentrations of cisplatin and other drug extravasation; Usage: Take $4 \mathrm{ml}$ of sodium thiosulfate mixed with $6 \mathrm{ml}$ of water for injection, subcutaneous injection at the site of extravasation, several hours later repeat.

\section{Conclusion}

As one of the important means for the treatment of malignant tumors, chemotherapy has become more and more widely used, and the risk of tissue damage caused by extravasation of chemotherapy drugs is also increasing. If not handled properly, it will not only directly affect the patient's treatment effect and quality of life, but also cause medical care disputes. Therefore, prevention and treatment of extravasation of chemotherapy drugs is of great significance. Nursing staff must pay enough attention to it, strengthen their sense of responsibility, strengthen specialist knowledge training, actively participate in clinical research, and determine the best nursing measures for extravasation of chemotherapy drugs. Through effective nursing interventions, early prevention, early detection, and early treatment can be achieved, which significantly reduces the extravasation rate of chemotherapy drugs, alleviates the pain caused by extravasation of chemotherapy drugs, and increases the trust between patients and nurses. Sense and satisfaction, thereby improving the safety and quality of care.

\section{Acknowledgements}

The fund project was included in the Qiqihar City Bureau of Science and Technology Bureau's instructional research project in 2015 (item number: SFZD-2015025)

\section{References}

[1] Zhang Qingxin, Xin Fukui, Li Hong. Protection of chemotherapy drug extravasation [J]. Journal of Chinese Journal of Misdiagnosis, 2011,11 (17): 4270.

[2] Ruan Yongyi, Zeng Yuanli, Zhong Ping. Progress in the treatment and care of tissue injury caused by chemotherapy drug extravasation[J]. Nursing Journal of Adult Nursing, 2009, 24(7): 617.

[3] Zhou Yinping, Chemotherapy, Extravasation, Protection, Progress [J], Qi Lu, Nursing Journal, 2012, 18(31): 46.

[4] Yaojie Yao, Rongxue Xie, Yanhua Huang. Prevention and treatment of chemotherapy drug extravasation [J]. General nursing, 2011, 9 (2): 298.

[5] Yang Hua.Special requirements for infusion of chemotherapy drugs and nursing strategies for extravasation [J]. Gansu medicine, 2012, 31(5): 392. 